\title{
Curing glioblastoma: oncolytic HSV-IL12 and checkpoint blockade
}

\section{Dipongkor Saha, Robert L. Martuza, and Samuel D. Rabkin}

Cancer immunotherapy has recently revolutionized how we approach the treatment of cancers, including fatal glioblastoma (GBM), but still fails to effectively treat the majority of cancer patients [1]. There are several key factors that may contribute to therapy failure, including, but not limited to: (i) low mutational loads and poor tumor immunogenicity; (ii) immune suppressive tumor microenvironment (regulatory $\mathrm{T}$ cells, pro-tumoral M2-like macrophages); and (iii) tumor heterogeneity, including therapy-resistant GBM stem cells (GSCs). GSCs contribute to tumor initiation, progression, maintenance, and recurrence, and are thus critical targets for therapy. Recently, we described a new stringent difficult-to-treat stem cell-based immune competent GBM model (005 GSC) that addresses aforementioned features of therapeutic hindrance: low mutational load with only two known somatic mutations; relatively nonimmunogenic, lacking MHCI and II expression, with PD-L1 only expressed on a minority of 005 GSCs; highly tumorigenic and invasive; and an immune suppressive tumor microenvironment $[2,3]$. This model has been used to test immunotherapeutic strategies for GBM.

Oncolytic viruses [4], e.g. oncolytic herpes simplex virus (oHSV), are a distinct class of anticancer agent with unique mechanisms of action: selective targeting and killing of cancer cells irrespective of tumor mutational load, immune status, and heterogeneity, while sparing normal cells, and exposing viral/tumor antigens, which promote cascades of anti-tumor (and anti-viral) immune responses (in situ vaccine) [5]. Despite these properties, oHSV G47 $\Delta$, which is currently in clinical trial in Japan, was insufficient alone in the 005 GSC model. However, viral expression of IL12 (G47D-mIL12) produced a significant but modest improvement in survival of 005 tumor-bearing mice $[2,3]$. This was associated with an increased number of tumor infiltrating $\mathrm{T}$ cells, increased effector/regulatory $\mathrm{T}$ cell ratio, skewing the tumorassociated macrophages (TAMs) towards an anti-tumoral M1-phenotype, and decreased tumor cells (Figure 1) [3].

Immune checkpoints, such as cytotoxic $\mathrm{T}$ lymphocyte antigen 4 (CTLA-4) and programmed death 1 (PD-1) play a critical role in regulating immune responses and suppressing immune effector cells. PD-1 and CTLA4 are expressed predominantly on T-cells, while PD-L1 is also expressed on endothelial and tumor cells. Blocking antibodies to these molecules are effective at reversing tumor-induced immunosuppression [1]. Therefore, we hypothesized that G47 $\Delta$-mIL12, which induces antitumor immune responses, should synergize with immune checkpoint inhibitor (ICI) antibodies in impeding GBM growth. In the 005 model, ICI antibody alone (anti-PD-1 or anti-CTLA-4) produced only modest improvement in

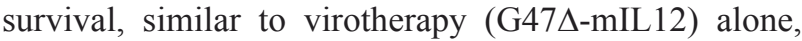
while the combination of G47A-mIL12 with either antibody or combination of two antibodies further extended survival modestly [3]. The limited efficacy of the dual combination was not due to the inability of antibodies to cross the blood brain/tumor barrier, since antibodies were detected in the tumor. However, the triple combination of G47 $\Delta$-mIL12+anti-CTLA-4+antiPD-1 cured $89 \%$ mice with 005 tumors and protected them from lethal tumor re-challenge. These findings were reproduced in another aggressive immune competent glioma model, CT-2A [3]. Though single or dual therapies significantly modulated the tumor microenvironment, triple combination therapy produced the most prominent anti-tumor effects, such as a significant reduction in tumor cells, influx of M1-like TAMs, increased proliferating $\mathrm{T}$ cells, and an increased $\mathrm{T}$ effector/regulatory cell ratio (Figure 1). Depletion studies demonstrated a requirement for $\mathrm{CD}^{+}, \mathrm{CD} 8^{+} \mathrm{T}$ cells, and macrophages for therapeutic efficacy, with $\mathrm{CD}^{+} \mathrm{T}$ cells playing an essential role [3]. It remains to be determined which factor/gene(s) were responsible for the complex immune cell interactions and how they contributed to $\mathrm{CD}^{+} \mathrm{T}$ cell-dependent therapeutic benefit. Whether triple therapy using other oncolytic viruses results in similar curative benefits will be important to determine.

An oHSV encoding human granulocyte-macrophage colony-stimulating factor (GM-CSF) (Talimogene laherparepvec or T-VEC), similar to G47 $\Delta$-mIL12, was recently approved for the treatment of patients with advanced melanoma, an immunogenic tumor [6]. Followon clinical trials with T-VEC in combination with antiCTLA-4 (ipilimumab) in melanoma elicited significant clinical responses, with a durable response rate of 50\% [7]. However, triple combination therapy (oHSV+anti-CTLA4+anti-PD-1) may be required to obtain curative responses in the majority of cancer patients with non-immunogenic or ICI non-responding tumors, as described for GBM [3].

An important issue to be addressed before the full potential of oHSV-based cancer immunotherapy is 
realized is maximizing oHSV replication/spread within the tumor and developing representative preclinical models. For example, oHSV replication is limited in mouse tumors [2], and anti-viral immune responses can limit virus spread in patients. Therefore, developing strategies to enhance tumor-specific viral replication and spread, and anti-tumor immunity without compromising safety is key for clinical success. More research is needed to optimize new viral vectors and design more rationale combination clinical trials. This may include the generation of new oHSV vectors expressing other immune modulators, testing them in combination with other immunotherapies, and expanding clinical development to patients with minimally immunotherapy responsive lethal cancers, like GBM.

\section{CONFLICTS OF INTEREST}

The authors declare no conflicts of interest.

Dipongkor Saha: Molecular Neurosurgery Laboratory and the Brain Tumor Research Center, Department of Neurosurgery, Massachusetts General Hospital and Harvard Medical School, Boston, MA, USA

Correspondence: Dipongkor Saha, email dipongkor.saha@ mgh.harvard.edu

Keywords: oncolytic virus, herpes simplex virus, cancer stem cells, immunotherapy, immune checkpoint

Received: September 05, 2017

Published: September 20, 2017

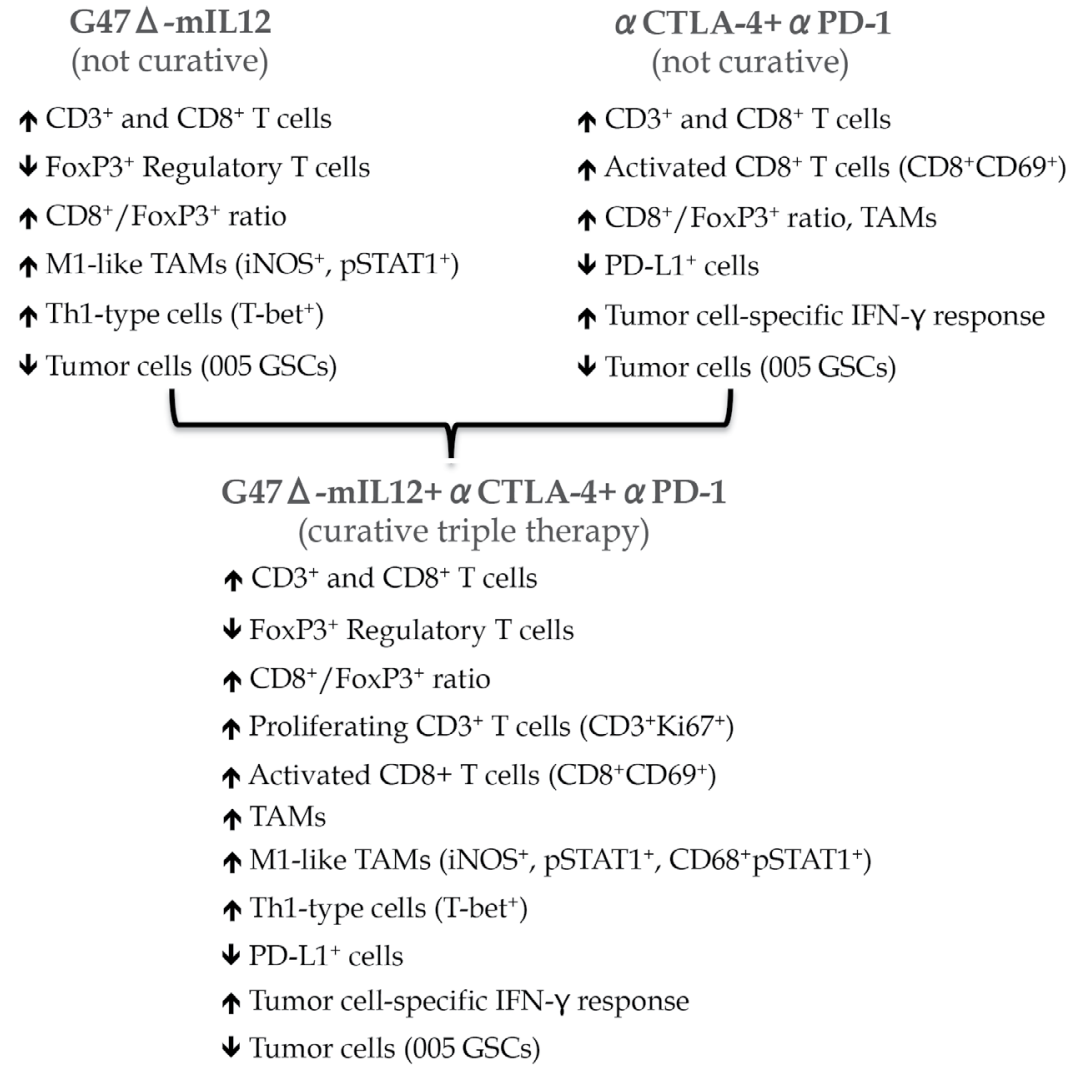

Figure 1. Treatment alterations in tumor microenvironment 


\section{REFERENCES}

1. Topalian SL, et al. Nat Rev Cancer. 2016; 16:275-87.

2. Cheema TA, et al. Proc Natl Acad Sci U S A. 2013; 110:12006-11.

3. Saha D, et al. Cancer Cell. 2017; 32:253-67 e5.

4. Saha D, et al. Drugs Future. 2015; 40:739-49.

5. Saha D, et al. Curr Opin Virol. 2016; 21:26-34.

6. Andtbacka RH, et al. J Clin Oncol. 2015; 33:2780-8.

7. Puzanov I, et al. J Clin Oncol. 2016; 34:2619-26.

Copyright: Saha et al. This is an open-access article distributed under the terms of the Creative Commons Attribution License 3.0 (CC BY 3.0), which permits unrestricted use, distribution, and reproduction in any medium, provided the original author and source are credited. 